\title{
OPEN Neurocognitive status and risk of mortality among people living with human immunodeficiency virus: an 18-year retrospective cohort study
}

Zaeema Naveed ${ }^{1}$, Howard S. Fox ${ }^{2}$, Christopher S. Wichman ${ }^{3}$, Morshed Alam ${ }^{3}$, Pamela May ${ }^{2}$, Christine M. Arcari ${ }^{1}$, Jane Meza ${ }^{3}$, Steven Totusek ${ }^{2} \&$ Lorena Baccaglini $^{1 凶}$

HIV-related neurocognitive impairment $(\mathrm{NCl})$ may increase the risk of death. However, a survival disadvantage for patients with $\mathrm{NCl}$ has not been well studied in the post-combination antiretroviral therapy (cART) era. Specifically, limited research has been conducted considering the reversible nature and variable progression of the impairment and this area demands further evaluation. We performed multivariable Cox proportional hazards modeling to assess the association between baseline $\mathrm{NCl}$ (global T scores) and mortality. A joint modeling approach was then used to model the trajectory of global neurocognitive functioning over time and the association between neurocognitive trajectory and mortality. Among the National NeuroAIDS Tissue Consortium's (NNTC) HIV-infected participants, we found a strong negative association between $\mathrm{NCl}$ and mortality in the older age groups (e.g., at age $=55, \mathrm{HR}=0.79 ; 95 \% \mathrm{Cl} 0.64-0.99$ ). Three neurocognitive sub-domains (abstraction and executive functioning, speed of information processing, and motor) had the strongest negative association with mortality. Joint modelling indicated a $33 \%$ lower hazard for every 10 -unit increase in global T scores $(\mathrm{HR}=0.67 ; 95 \% \mathrm{Cl} 0.56-0.80)$. The study identified older HIV-infected individuals with $\mathrm{NCl}$ as a group needing special attention for the longevity of life. The study has considerable prognostic utility by not only predicting mortality hazard, but also future cognitive status.

Since the outset of the human immunodeficiency virus (HIV) epidemic, HIV-associated neurocognitive disorders (HAND) have been prevalent in infected populations, ranging from subtle neuropsychological impairments to profoundly disabling HIV-associated dementia (HAD) ${ }^{1}$. The advent of combination antiretroviral therapy (cART) has transformed HIV infection from a deadly acute disease to a chronic tractable condition by effective management of HIV viremia and enhanced immune function ${ }^{2}$. Yet, despite the widespread use of cART, HIV-associated neurocognitive impairment (NCI) and brain injury persist with a change in phenotype and pattern. There has been a significant decrease in HAD in the cART era. Nevertheless, less severe forms of HAND continue to have a prevalence of $20-50 \%^{3,4}$. The pattern of NCI also differs between the two eras. In the pre-cART era impairment in motor skills, cognitive speed, and verbal fluency were more common, whereas in the cART era memory and executive function impairment are more prominent ${ }^{1,3,5}$.

HIV enters the brain early in its course by crossing the blood-brain barrier inside migrating monocytes and lymphocytes. Infected monocytes are converted to perivascular macrophages that express neurotoxic molecules leading to increased blood-brain barrier permeability. Neuronal damage and death are ensued both by direct viral proteins interaction and indirect inflammatory response mounted by inflammatory cells against the viral proteins $^{6,7}$. The brain alterations during early HIV infection have also been validated by neuroimaging ${ }^{8}$. In the past, subcortical regions of the brain were thought to be primarily infected by HIV, giving rise to subcortical dementia. However, heterogeneous findings from both neuropsychological and neuroimaging studies have now recognized the cognitive impairment to be present across various brain regions and cognitive domains ${ }^{9}$. The

${ }^{1}$ Department of Epidemiology, College of Public Health, University of Nebraska Medical Center, 984355 Medical Center, Omaha, NE 68198-4395, USA. ${ }^{2}$ Department of Neurological Sciences, University of Nebraska Medical Center, Omaha, NE, USA. ${ }^{3}$ Department of Biostatistics, University of Nebraska Medical Center, Omaha, NE, USA. ${ }^{\varpi}$ email: lorena.baccaglini@unmc.edu 
exact mechanism of persistence of milder forms of impairment is not clear; though, two potential explanations may include the lingering consequences of advanced immunosuppression during the early stages of the disease (before initiation of cART) and ongoing viral replication within the brain, even when systemic viral suppression has been achieved ${ }^{10,11}$.

NCI progression is highly variable, with individuals displaying considerable recovery of cognitive functions, worsening of impairment, static impairment, or a fluctuating course ${ }^{12}$. Understanding the consequences of HIV associated NCI is vital because even in milder forms, it is associated with lower medication adherence, a decreased ability to perform the daily tasks, poorer quality of life, and difficulty obtaining employment ${ }^{13}$. Moreover, HIV infected individuals with mild cognitive impairment may have an increased risk of dementia and death ${ }^{14,15}$. Although HIV related morbidity and mortality have decreased over time, people living with HIV continue to face an increased risk of mortality compared to the non-infected counterparts, even among those with a successful response to $\mathrm{CART}^{16,17}$. There are several well-established predictors of mortality in HIV ${ }^{18-20}$; however, limited research has been conducted to investigate the association between NCI and mortality in HIV infected people. In the pre-cART era, NCI ascertained through a comprehensive battery of neuropsychological tests was found to be an independent risk factor of death ${ }^{15,21,22}$. A recent cross-sectional study with hospitalized HIV infected patients as the study sample found higher inpatient mortality among those who had been diagnosed with HIV associated NCI compared to those who had not ${ }^{23}$. Three other studies conducted in the cART era found a positive association between NCI and mortality but were limited to participants with advanced HIV infection or severe cognitive disorders only ${ }^{24-26}$. Banerjee et al. recently reported NCI to be an independent prognostic marker of mortality in an HIV infected cognitive cohort ${ }^{27}$. The study used the HIV-Dementia scale (HDS) to assess NCI. However, studies have demonstrated inconsistent results pertaining to the ability of HDS to detect subtle types of $\mathrm{NCI}^{28,29}$.

Apart from the limited research on the association between NCI and mortality, all the previous studies have examined cognitive impairment at a single time-point (i.e., baseline). As it is likely that cognitive status changes over time, it is vital to account for this variability in relation to mortality. A survival disadvantage for patients with NCI has not been well studied in the cART era, particularly taking into consideration the reversible nature of the impairment and demands further evaluation. The present study aims to fill the research gap by examining the association between baseline neurocognitive status as well as longitudinal changes in neurocognitive status and mortality in a diverse HIV-infected sample. We hypothesize that NCI and its progression increase the hazard of death in HIV patients either independently or in association with specific patient-related factors.

\section{Methods}

Data source and participants. The National NeuroAIDS Tissue Consortium (NNTC) database was used to investigate the association between neurocognitive status and mortality in HIV patients. NNTC is an ongoing, prospective observational study established in 1998 with the primary aim of collecting, storing, and distributing samples of central and peripheral nervous system tissue, cerebrospinal fluid, blood, and other organs collected from HIV positive and negative patients for research purposes ${ }^{30}$. Adult participants with advanced HIV disease willing to participate in a post-mortem organ donation program were recruited at one of the four participating sites: Texas NeuroAIDS Research Center (University of Texas Medical Branch, Galveston), California NeuroAIDS Tissue Network (University of California, San Diego), National Neurological AIDS Bank (University of California, Los Angeles) and Manhattan HIV Brain Bank (Mount Sinai Medical Center, New York). Participants were volunteers recruited from clinics, hospitals, and local communities into a longitudinal observational study with detailed neurologic and neuropsychological evaluations at 6-, 12- or 24-month intervals depending on the clinical judgment of a participant's health.

Variables such as demographics, medication history (ARV and others), cerebrospinal fluid, blood, plasma, and urine laboratory testing for HIV specific and ancillary markers, comorbidities and substance use were collected at baseline and during the longitudinal phase ${ }^{31,32}$.

For the analysis, we included participants enrolled between January 2000 to November 2017, with complete baseline data on the variables of interest, and with at least two follow-up visits $(n=1,325)$. Seventy-seven participants did not have information available on neurocognitive status at the baseline and were excluded $(n=1,248)$. Further exclusions were made based on missing baseline information on covariates of interest. The reporting of this observational study has been guided by the STROBE instrument.

Variables. The primary outcome was time to event (death). The primary exposures were neurocognitive status at baseline (for Cox proportional hazards modeling) and repeated measures of neurocognitive status (for joint modeling). Neurocognitive status was assessed through a continuous score (demographically corrected $\mathrm{T}$ score) derived from a comprehensive neurocognitive test battery comprising of fourteen test scores. The tests with references are given in Table 1 . The battery covers seven cognitive domains, including executive functioning, speed of information processing, attention and working memory, learning, memory, verbal fluency, and motor functioning. Raw scores from individual tests were converted to demographically corrected $\mathrm{T}$ scores ${ }^{3}$ which were than averaged together to generate the global T score. For descriptive analysis, an impaired neurocognitive status was assigned to those with a global T score value of $<40^{33}$. The best available normative standards were used, which correct for the effects of age, education, sex, and ethnicity, as appropriate ${ }^{14}$. Based on prior literature and biological plausibility, other groups of variables included in the study were demographic factors (age, education, gender, race, and ethnicity), HIV related factors (disease severity, duration of HIV infection, antiretroviral (ARV) drug use, CD4 nadir, current CD4 cell count, plasma viral loads and CSF viral loads), comorbidities (anemia, cerebrovascular disease, hypertension, diabetes, hyperlipidemia, viral hepatitis, chronic renal disease, chronic obstructive pulmonary disease, AIDS-defining comorbidity, any CNS comorbidity and a 


\begin{tabular}{|c|c|}
\hline Cognitive domain & Neuropsychological test \\
\hline \multirow{2}{*}{ Abstraction/executive functioning } & Trail Making Test, Part $\mathrm{B}^{49}$ \\
\hline & Wisconsin Card Sorting Test-64, Perseverative Responses ${ }^{49,50}$ \\
\hline \multirow{3}{*}{ Speed of information processing } & Wechsler Adult Intelligence Scale-3rd ed. (WAIS-III) Digit Symbol ${ }^{51}$ \\
\hline & WAIS-III Symbol Search ${ }^{51}$ \\
\hline & Trail Making Test, Part A ${ }^{49}$ \\
\hline \multirow{2}{*}{ Attention and working memory } & Paced Auditory Serial Addition Task (PASAT) (first channel only) ${ }^{52}$ \\
\hline & WAIS-III Letter Number Sequencing ${ }^{51}$ \\
\hline \multirow{2}{*}{ Learning } & Brief Visuospatial Memory Test-Revised (BVMT-R) Total Recall ${ }^{50,53}$ \\
\hline & Hopkins Verbal Learning Test-Revised (HVLT-R) Total Recall ${ }^{50,54}$ \\
\hline \multirow{2}{*}{ Memory } & BVMT-R Delayed Recall ${ }^{50,53}$ \\
\hline & HVLT-R Delayed Recall ${ }^{50,54}$ \\
\hline Verbal fluency & Controlled Oral Word Association Test (COWAT-FAS) ${ }^{49}$ \\
\hline \multirow{2}{*}{ Motor } & Grooved Pegboard dominant ${ }^{49}$ \\
\hline & Grooved Pegboard non-dominant ${ }^{49}$ \\
\hline
\end{tabular}

Table 1. Neuropsychological test battery used to generate T scores among HIV-infected participants of National NeuroAIDS Tissue Consortium (NNTC).

composite measure of any non-AIDS defining comorbidity) and substance use (history of alcohol, opiate, hallucinogen, cannabis and cocaine use). The Composite International Diagnostic Interview (CIDI, v2.1) that is consistent with the diagnostic and statistical manual of mental disorders-4th Edition (DSM-IV), was used to measure substance use.

Statistical analyses. Descriptive statistics were generated for categorical (frequencies and percentages) and continuous variables (means, medians, and standard deviations) to assess the overall demographic and clinical characteristics of the sample. Traditional survival analysis was conducted using Cox proportional hazards regression. Kaplan-Meier analysis was used to compute the overall median survival time and to visualize survival time distributions. Univariable Cox proportional hazards models were employed to explore the association between the covariates (primary exposure and potential confounders) and outcome. Furthermore, univariable analyses using one-way analysis of variance (ANOVA) for continuous variables and Cochran Mantel-Haenszel statistics (with modified ridit scores) for categorical variables were conducted to identify the association between primary covariate (neurocognitive status) and other potential covariates. Only those variables associated with, both with the primary outcome and primary covariate at 2 -sided $\alpha=0.2$, were entered in the multivariable Cox proportional hazards model for further analysis. One-way interactions between the primary predictor and other covariates were also assessed using the proportional hazards model.

For multivariable Cox proportional hazards modeling, a stepwise approach was used. The initial model included neurocognitive status, age, and the interaction term between them. Other potential confounders were then added to the model, and the change in the estimate for the association between neurocognitive status and outcome was recorded. The variable was considered a confounder if the percent change in the estimate of the reduced model compared to the model with the added variable was more than $5 \%$. Finally, HIV duration was forced into the model. The proportional hazards assumption was assessed using the graphical method (log-log survival curve approach) and goodness of fit test (based on martingale residuals). The same model was fitted for all seven domains as sub-analyses. Crude and adjusted hazard ratios (HR) and 95\% confidence intervals (95\% $\mathrm{CI}$ ) were reported as the measures of association for the Cox proportional hazards regression analyses. A subanalysis of participants with complete data on Beck Depression Inventory-II (BDI-II) scores was conducted to investigate potential confounding effects of depressive symptoms.

A joint modeling approach was then used to model the trajectory of global neurocognitive functioning (global $\mathrm{T}$ score) over time and the association between neurocognitive trajectory and mortality at the same time. The temporal evolution of longitudinal $\mathrm{T}$ score measurements was estimated using a linear mixed-effects model. To model cognitive trajectories, we employed both linear and quadratic functions to observe if the results differed. Residual diagnostics were conducted to check the linear mixed models' assumptions. The final joint model used a Weibull baseline hazard function and was adjusted for covariates used in the traditional survival analysis. The analyses were performed using SAS 9.4 (SAS Institute, Inc., Cary, NC, USA) software and R software-3.5.0 (JM package).

Ethical approval. This study was approved by the University of Nebraska Medical Center's institutional review board (Protocol \#282-13-EP). All methods were carried out in accordance with relevant guidelines and regulations. The National NeuroAIDS Tissue Consortium obtained written informed consent for participation, neuro-medical and neuropsychological examinations and for obtaining brain tissue after death for examination and tissue banking. For deceased participants, the decedent's family/legal guardian provided informed consent for tissue donation and release of medical records. 


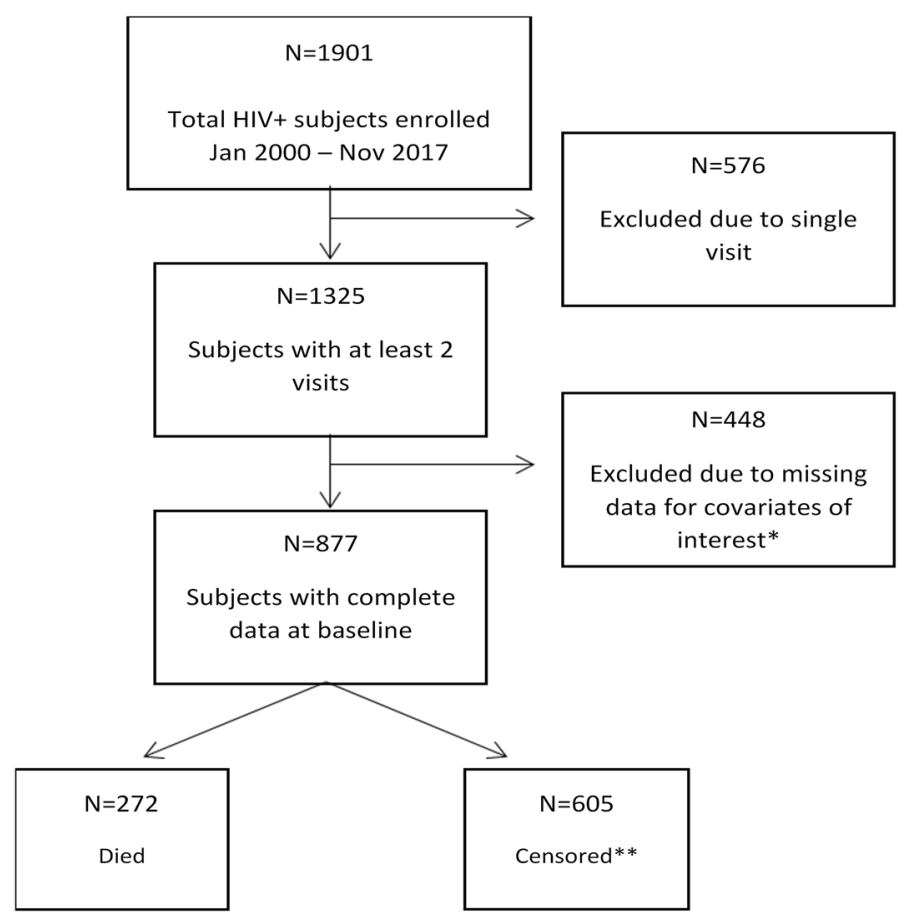

Figure 1. Flow chart of participation. ${ }^{\star}$ Descriptive analysis comparing those with missing data for covariates of interest to those with complete data showed no difference in demographic variables and baseline global $\mathrm{T}$ scores. ${ }^{*}$ There is a $12 \%$ loss to follow-up.

\section{Results}

Participants' characteristics. The original enrolled sample $(n=1,901)$ had a median age of 45 years $(\mathrm{IQR}=39-53)$, and was $81.1 \%$ males, $55.8 \%$ whites and $73.2 \%$ non-Hispanics. Fifty nine percent of the original sample had a global T score above 40 . A total of 877 participants were included in the final analyses (Fig. 1). The median age was 45 years (IQR $=40-53)$, and most participants were males $(79.2 \%)$, whites $(55.8 \%)$ and nonHispanics/Latinos (72.1\%). Sixty percent of participants in the analytic sample had a global T score above 40 . The median duration of HIV infection was 12.6 years (IQR $=7.4-17.8)$. At baseline, $78 \%$ of participants were on a highly active antiretroviral therapy (HAART) regimen, $13 \%$ were on the non-HAART regimen, and the rest $9 \%$ were not using any antiretroviral therapy (ART). The median CD4+cell count was 203.5 cells/ $\mu \mathrm{l}(\mathrm{IQR}=75-406)$ and the median $\log _{10}$ viral load was $2.6(\mathrm{IQR}=1.7-4.2)$. Table 2 shows the baseline characteristics of participants by neurocognitive status. Most males (80.5\%), participants on HAART (81.1\%), Blacks (42.0\%), and nonHispanics/Latinos (77.4\%) were in the neurocognitively unimpaired category. Furthermore, participants in the unimpaired category were marginally older ( mean $=47.1$ years) with higher education ( mean $=12.3$ years), higher hemoglobin levels $($ mean $=13.4 \mathrm{~g} / \mathrm{dl})$, higher CD4 count $($ mean $=301.9$ cells $/ \mu \mathrm{l})$, lower blood $\log _{10}$ viral load (mean $=2.9$ copies $/ \mathrm{ml}$ ), longer duration of HIV disease (mean $=13.3$ years) and fewer CNS comorbidities (mean $=0.05$ ) compared to those in impaired group. Descriptive statistics for the neurocognitive battery of tests (individual and domain specific T tests) are included in Supplementary Table S1. Participants that died during the follow-up had marginally lower baseline $\mathrm{T}$ scores for the domains of abstraction/executive functioning, speed of information processing, verbal fluency and motor and marginally higher $\mathrm{T}$ scores for the domains of attention and working memory, learning and working memory compared to those that were censored.

Survival analysis (Kaplan-Meier method and Cox proportional hazards regression). The participants were followed for up to 18 years. During the study duration, of the 877 participants, 272 (31\%) died. The overall median survival time was 13.2 years (Fig. 2). The survival plot (Fig. 3) stratified by neurocognitive status, exhibited lower median survival time (median $=11.8$ years) for neurocognitively impaired participants compared to the median survival time (14.1 years) for unimpaired participants. Unadjusted analyses for interaction and identification of potential confounders showed a significant interaction between age and neurocognitive status in association with mortality. Furthermore, 11 variables (current ARV medication use, ethnicity, history of hyperlipidemia, history of cerebrovascular disease, history of cannabis use, history of cocaine use, history of opiate use, serum hemoglobin, CD4 nadir, plasma viral loads and duration of HIV infection) were assessed as potential confounders in a stepwise approach in the adjusted model. The final multivariable Cox proportional hazards model included duration of HIV infection, ethnicity, serum hemoglobin, plasma viral load and an interaction term between neurocognitive status and age (Table 3). The adjusted model revealed a strong negative association between mortality and NCI in the older age groups. For example, among those who were 55 years old, the hazard of dying for those with higher ( 10 units) global T score was 0.79 times ( $21 \%$ lower) the hazard for those with lower global T score. However, the association was not evident in the younger age groups. 


\begin{tabular}{|c|c|c|c|}
\hline \multirow[b]{3}{*}{ Variables } & \multicolumn{2}{|l|}{ Global $T$ scores $^{\mathrm{a}}$} & \multirow[b]{3}{*}{$p$ value } \\
\hline & Unimpaired $>40$ & \multirow{2}{*}{$\begin{array}{l}\text { Impaired } \leq 40 \\
\mathrm{n}(\%)^{\mathrm{b}}\end{array}$} & \\
\hline & n (\%) & & \\
\hline Total & $527^{c}(60.1)$ & $350^{c}(39.1)$ & \\
\hline \multicolumn{4}{|l|}{ Gender } \\
\hline Male & $424(80.5)$ & $271(77.4)$ & $0.2791^{*}$ \\
\hline ARV medication use & & & $0.0157^{*}$ \\
\hline No current ARV & $45(9.1)$ & $29(8.9)$ & \\
\hline Current non-cART & $49(9.8)$ & $59(18.0)$ & \\
\hline Current cART & $402(81.1)$ & $239(73.1)$ & \\
\hline Race & & & $0.003^{*}$ \\
\hline White & $266(51.9)$ & $211(61.7)$ & \\
\hline Black & $215(42.0)$ & $98(28.6)$ & \\
\hline Other & $33(9.6)$ & $33(9.6)$ & \\
\hline Ethnicity & & & $<0.0001^{*}$ \\
\hline Hispanic or Latino & $119(22.6)$ & $126(36.0)$ & \\
\hline Not Hispanic or Latino & $408(77.4)$ & $224(64.0)$ & \\
\hline Hypertension history & $121(29.9)$ & $62(25.4)$ & $0.2138^{*}$ \\
\hline Diabetes history & $46(11.39)$ & $43(17.7)$ & $0.0242^{*}$ \\
\hline Hyperlipidemia history & $100(24.7)$ & $48(19.6)$ & $0.1291^{*}$ \\
\hline Viral hepatitis history & $139(34.5)$ & $104(42.8)$ & $0.0340^{*}$ \\
\hline End stage liver disease history & $9(2.2)$ & $8(3.3)$ & $0.4157^{*}$ \\
\hline Chronic renal disease history & $30(7.5)$ & $17(7.0)$ & $0.8252^{*}$ \\
\hline Cardiac disease history & $40(9.9)$ & $26(10.7)$ & $0.7611^{*}$ \\
\hline Chronic obstructive pulmonary disease & $44(10.9)$ & $24(9.8)$ & $0.6563^{*}$ \\
\hline Cerebrovascular disease history & $33(8.2)$ & $43(17.7)$ & $0.0005^{*}$ \\
\hline Non-AIDS defining cancers & $31(7.7)$ & $15(6.2)$ & $0.4622^{*}$ \\
\hline Any non-AIDS defining comorbidity ${ }^{\mathrm{d}}$ & $287(70.7)$ & $181(73.9)$ & $0.3811^{*}$ \\
\hline Any CNS comorbidity ${ }^{e}$ & $29(5.9)$ & $44(13.4)$ & $0.0002^{*}$ \\
\hline Alcohol use history & $224(61.5)$ & $98(45.5)$ & $0.0030^{*}$ \\
\hline Cannabis use history & $151(41.5)$ & $62(28.6)$ & $0.0379^{*}$ \\
\hline Cocaine use history & $198(54.4)$ & $87(40.1)$ & $0.0008^{*}$ \\
\hline Hallucinogen use history & $28(7.7)$ & $16(7.4)$ & $0.8883^{*}$ \\
\hline Opiate use history & $63(17.3)$ & $42(19.3)$ & $0.5345^{\star}$ \\
\hline Sedative use history & $38(10.4)$ & $24(11.1)$ & $0.8149^{*}$ \\
\hline \multirow[t]{2}{*}{ Stimulant use history ${ }^{e}$} & $86(23.6)$ & $39(17.9)$ & $0.1089^{*}$ \\
\hline & Mean (SD) & Mean (SD) & \\
\hline Age (years) & $47.1(10.7)$ & $46.5(10.6)$ & $0.5779^{* *}$ \\
\hline Years of education (years) & $12.3(3.6)$ & $12.2(3.2)$ & $0.5779^{* *}$ \\
\hline Beck's Depression Inventory (BDI) total score & $13.1(10.1)$ & $15.4(10.4)$ & $0.0001^{* *}$ \\
\hline Hemoglobin (g/dl) & $13.4(1.8)$ & $13.2(1.9)$ & $0.0395^{* *}$ \\
\hline CD4 Nadir (cells/ $\mu$ l) & $138.7(213.6)$ & $138.1(210.0)$ & $0.9744^{* *}$ \\
\hline CD4 cell count (cells/ $\mu \mathrm{l})$ & $301.9(298.8)$ & $273.1(273.5)$ & $0.1565^{* *}$ \\
\hline Plasma viral load (log10 copies/ml) & $2.9(1.3)$ & $3.1(1.4)$ & $0.0561^{* *}$ \\
\hline Number of non-AIDS defining comorbidities ${ }^{d}$ & $1.4(1.3)$ & $1.6(1.5)$ & $0.2521^{* *}$ \\
\hline Number of CNS comorbidities ${ }^{\mathrm{f}}$ & $0.05(0.2)$ & $0.1(0.4)$ & $<.0001^{* *}$ \\
\hline Duration of HIV (years) & $13.3(7.4)$ & $12.7(7.6)$ & $0.1615^{* *}$ \\
\hline Number of substances used ${ }^{g}$ & $2.1(1.7)$ & $1.6(1.7)$ & $0.0323^{* *}$ \\
\hline
\end{tabular}

Table 2. Participant's baseline characteristics by neurocognitive status among HIV-infected participants of National NeuroAIDS Tissue Consortium (NNTC). ${ }^{\star}$ Cochran Mantel-Haenszel statistics (score $=$ modified

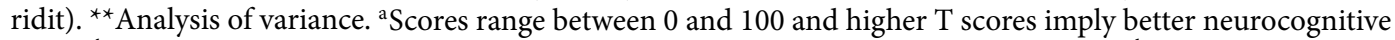
status. ${ }^{b}$ Column percentages. ${ }^{c}$ Sample size may be lower for variables due to missing data. ${ }^{\mathrm{d}}$ Includes history of hypertension, diabetes, viral hepatitis, end-stage liver disease, hyperlipidemia, chronic renal disease, cardiac disease, chronic obstructive pulmonary disease, and cerebrovascular disease. ${ }^{~}$ Defined as use of amphetamines,

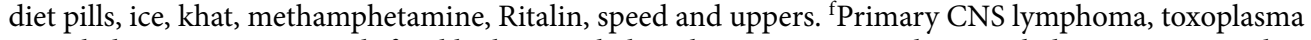
encephalitis, progressive multifocal leukoencephalopathy, CMV ventriculo-encephalitis, cryptococcal meningitis, histoplasma meningitis, coccidiodes meningitis, tuberculous meningitis, syphilitic meningitis, lymphomatous meningitis, and other specific meningitis. 'Includes history of alcohol, cannabis, cocaine, hallucinogen, opiate, sedative and stimulant use. 


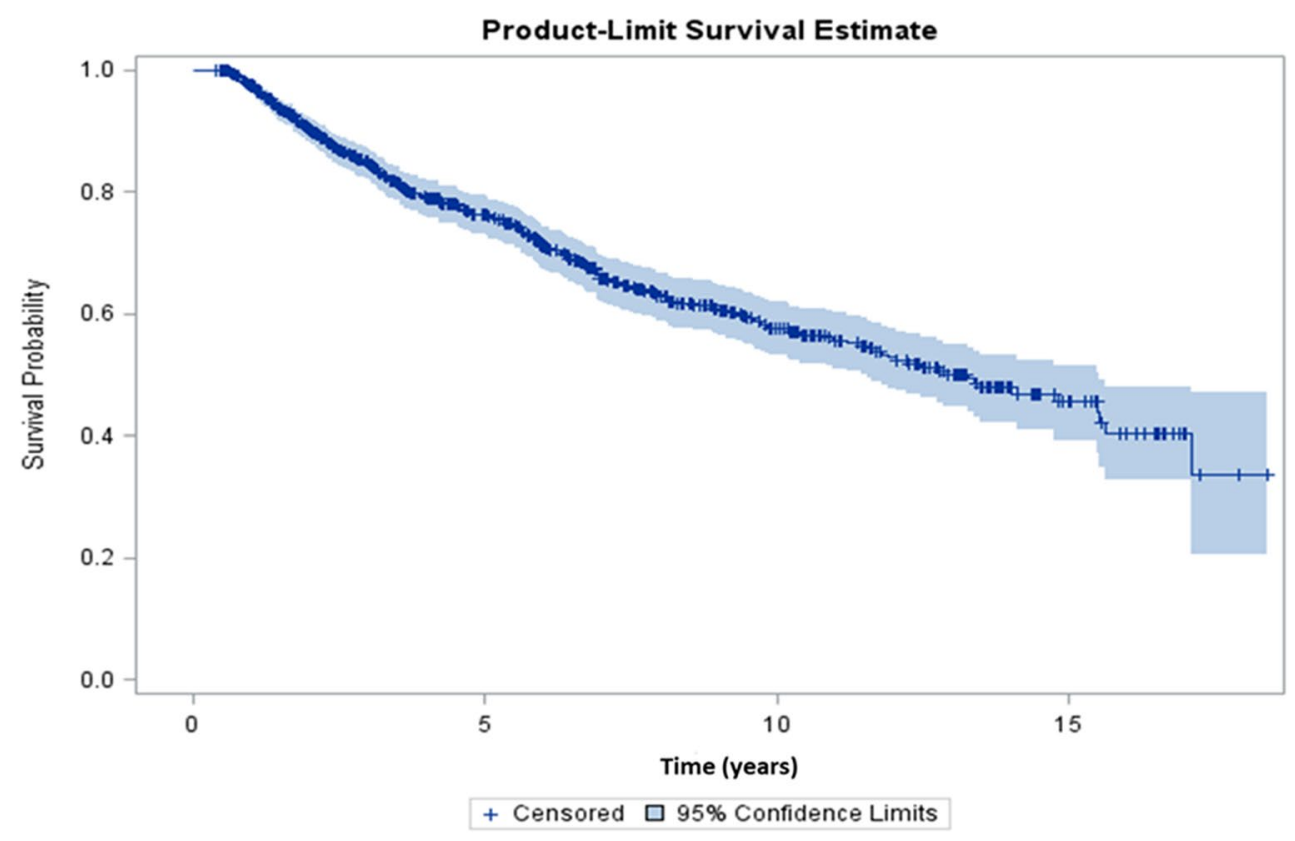

Figure 2. Kaplan-Meier plot for overall survival. Median survival time $=13.2$ years (95\% CI 11.4-15.5).

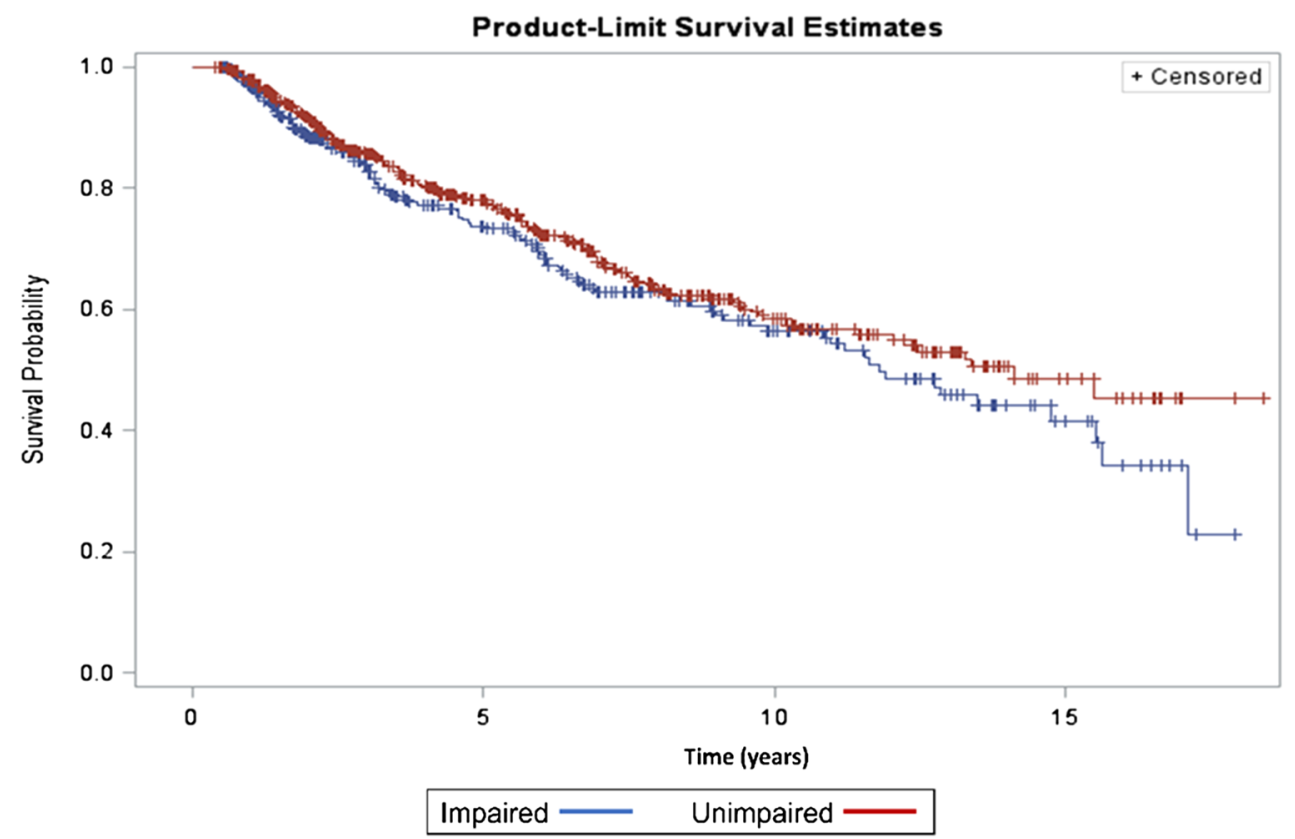

Figure 3. Kaplan-Meier plot stratified by neurocognitive status. Median survival time impaired (global $\mathrm{T}$ score $\leq 40)=11.8$ years $(95 \%$ CI 9.6-15.5). Median survival time unimpaired (global T score $>40)=14.1$ years (95\% CI 9.6). ${ }^{*}$ The upper limit of confidence interval for median survival time in unimpaired group is not estimable because of censored data.

Apart from the interaction term, being non-Hispanic/Latino (HR=1.38, 95\% CI 1.02-1.86) and having a higher baseline $\log 10$ viral load ( $\mathrm{HR}=1.27,95 \% \mathrm{CI} 1.16-1.39)$ were associated with a higher hazard of dying whereas, a higher baseline serum hemoglobin was associated with a lower hazard of dying ( $\mathrm{HR}=0.91,95 \%$ CI 0.85-0.97). Domain-specific Cox proportional hazards modeling (Table 4) showed that T scores for abstraction and executive functioning, speed of information processing, and motor domains, had stronger negative associations with mortality compared to the other domains, particularly among older participants. As seen with the global neu- 


\begin{tabular}{|c|c|c|}
\hline Variable (at baseline) & Crude HR (95\% CI) & Adjusted HR $(95 \% \mathrm{CI})$ model $^{\mathrm{a}}$ \\
\hline Global T score ${ }^{\mathrm{b}}$ & $0.99(0.97-1.01)$ & NA \\
\hline Age (years) & $1.01(0.99-1.02)$ & NA \\
\hline \multicolumn{3}{|l|}{ Gender } \\
\hline Female & Ref & \multirow{2}{*}{ NA } \\
\hline Male & $1.20(0.90-1.61)$ & \\
\hline \multicolumn{3}{|l|}{ Race } \\
\hline White & Ref & \multirow{3}{*}{ NA } \\
\hline Black & $1.15(0.89-1.48)$ & \\
\hline Other & $1.02(0.63-1.66)$ & \\
\hline Duration of HIV infection (years) & $1.02(1.01-1.03)$ & $1.01(0.99-1.03)$ \\
\hline \multicolumn{3}{|l|}{ Ethnicity } \\
\hline Hispanic or Latino & Ref & Ref \\
\hline Not Hispanic or Latino & $1.41(1.05-1.88)$ & $1.38(1.02-1.86)$ \\
\hline \multicolumn{3}{|l|}{ ARV medication use } \\
\hline No current ARV use & Ref & \multirow{3}{*}{ NA } \\
\hline Current non-cART & $0.52(0.32-0.86)$ & \\
\hline Current cART & $0.53(0.37-0.76)$ & \\
\hline Serum hemoglobin $(\mathrm{g} / \mathrm{dl})$ & $0.89(0.83-0.95)$ & $0.91(0.85-0.97)$ \\
\hline Plasma viral load (log10 copies/ml) & $1.28(1.17-1.39)$ & $1.27(1.16-1.39)$ \\
\hline \multicolumn{3}{|l|}{ T scores $\times$ age $^{c}$} \\
\hline 35 years & $1.13(0.88-1.46)$ & $1.09(0.85-1.41)$ \\
\hline 55 years & $0.76(0.61-9.95)$ & $0.79(0.64-0.99)$ \\
\hline 75 years & $0.51(0.31-0.85)$ & $0.58(0.35-0.97)$ \\
\hline
\end{tabular}

Table 3. Crude and adjusted hazard ratios (HR) for mortality by selected variables among HIV-infected

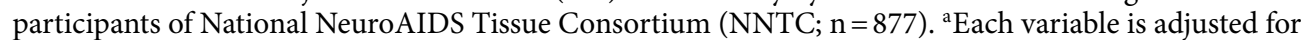
all other variables listed in the column. ${ }^{b}$ Hazard Ratio corresponds to a 10 -unit increase in global-t scores. ${ }^{\mathrm{c}}$ Interaction term between global $\mathrm{T}$ scores and age. Hazard ratio corresponds to a 10 -unit increase in global $\mathrm{T}$ scores for a given age.

\begin{tabular}{|c|c|c|c|c|c|c|c|c|}
\hline \multirow[b]{2}{*}{$\begin{array}{l}\text { Variable (at } \\
\text { baseline) }\end{array}$} & \multirow[b]{2}{*}{$\begin{array}{l}\text { Global T score } \\
\text { Adjusted HR (95\% } \\
\text { CI })^{\mathrm{a}}\end{array}$} & \multicolumn{7}{|c|}{$\begin{array}{l}\text { Domain specific T score } \\
\text { Adjusted HR }(95 \% \mathrm{CI})^{\mathrm{a}}\end{array}$} \\
\hline & & \begin{tabular}{|l|} 
Abstraction \\
executive \\
functioning \\
\end{tabular} & \begin{tabular}{|l} 
Speed of \\
information \\
processing
\end{tabular} & Motor & $\begin{array}{l}\text { Attention and } \\
\text { working memory }\end{array}$ & Learning & Memory & Verbal fluency \\
\hline $\mathrm{T}$ scores $^{\mathrm{b}}$ & NA & NA & NA & NA & NA & NA & NA & NA \\
\hline Age (years) & NA & NA & NA & NA & NA & NA & NA & NA \\
\hline $\begin{array}{l}\text { Duration of HIV } \\
\text { infection (years) }\end{array}$ & $1.01(0.99-1.03)$ & $1.01(0.99-1.03)$ & $1.01(0.99-1.03)$ & $1.01(0.99-1.03)$ & $1.01(0.99-1.03)$ & $1.01(0.99-1.03)$ & $1.01(0.99-1.03)$ & $1.01(0.99-1.03)$ \\
\hline \multicolumn{9}{|l|}{ Ethnicity } \\
\hline Hispanic or Latino & Ref & Ref & Ref & Ref & Ref & Ref & Ref & Ref \\
\hline $\begin{array}{l}\text { Not Hispanic or } \\
\text { Latino }\end{array}$ & $1.38(1.02-1.86)$ & $1.37(1.01-1.84)$ & $1.35(0.99-1.82)$ & $1.33(0.99-1.79)$ & $1.29(0.95-1.74)$ & $1.33(0.99-1.80)$ & $1.29(0.96-1.74)$ & $1.35(1.00-1.81)$ \\
\hline $\begin{array}{l}\text { Serum hemoglobin } \\
(\mathrm{g} / \mathrm{dl})\end{array}$ & $0.91(0.85-0.97)$ & $0.91(0.85-0.97)$ & $0.91(0.85-0.97)$ & $0.91(0.85-0.97)$ & $0.89(0.84-0.96)$ & $0.90(0.84-0.96)$ & $0.90(0.84-0.96)$ & $0.90(0.85-0.97)$ \\
\hline $\begin{array}{l}\text { Plasma viral load } \\
(\log 10 \text { copies } / \mathrm{ml})\end{array}$ & $1.27(1.16-1.39)$ & $1.27(1.16-1.39)$ & $1.27(1.16-1.39)$ & $1.28(1.16-1.40)$ & $1.28(1.17-1.41)$ & $1.27(1.16-1.39)$ & $1.27(1.16-1.39)$ & $1.25(1.14-1.37)$ \\
\hline \multicolumn{9}{|l|}{ T scores $\times$ age $^{c}$} \\
\hline 35 years & $1.09(0.85-0.41)$ & $1.08(0.90-1.30)$ & $1.09(0.90-1.33)$ & $0.98(0.84-1.14)$ & $0.99(0.80-1.24)$ & $1.11(0.89-1.37)$ & $1.16(0.95-1.41)$ & $0.99(0.82-1.18)$ \\
\hline 55 years & $0.79(0.64-0.99)$ & $0.83(0.71-0.97)$ & $0.84(0.71-0.98)$ & $0.80(0.68-0.94)$ & $0.95(0.79-1.14)$ & $0.93(0.77-1.12)$ & $1.01(0.85-1.20)$ & $0.89(0.77-1.03)$ \\
\hline 75 years & $0.58(0.35-0.97)$ & $0.64(0.45-0.93)$ & $0.64(0.43-0.94)$ & $0.65(0.45-0.94)$ & $0.91(0.59-1.40)$ & $0.78(0.50-1.20)$ & $0.88(0.59-1.32)$ & $0.81(0.57-1.14)$ \\
\hline
\end{tabular}

Table 4. Neurocognitive domain specific crude and adjusted hazard ratios for mortality by selected variables among HIV-infected participants of National NeuroAIDS Tissue Consortium (NNTC; $\mathrm{n}=877$ ). ${ }^{\mathrm{a}}$ Each variable is adjusted for all other variables listed in the column. ${ }^{\mathrm{b}} \mathrm{Hazard}$ Ratio correspond to a 10 -unit increase in $\mathrm{T}$ scores. ${ }^{\mathrm{c}}$ Interaction term between $\mathrm{T}$ scores and age. Hazard ratio corresponds to a 10 -unit increase in $\mathrm{T}$ scores for given age. 


\begin{tabular}{|l|l|l|}
\hline Predictor & $\boldsymbol{\beta}(\mathrm{SE})$ & ${\text { Adjusted HR (95\% CI })^{\mathbf{a}}}$ \\
\hline Intercept & $-2.34(0.71)$ & $0.09(0.02-0.38)$ \\
\hline Global T score slope & $-0.39(0.08)$ & $0.67^{\mathrm{a}}(0.56-0.80)$ \\
\hline Age (years) & $0.01(0.01)$ & $1.01(0.99-1.02)$ \\
\hline Duration of HIV infection (years) & $0.02(0.01)$ & $1.02(0.99-1.03)$ \\
\hline Ethnicity & \multicolumn{2}{|l|}{} \\
\hline Hispanic or Latino & Ref & Ref \\
\hline Not Hispanic or Latino & $0.41(0.15)$ & $1.50(1.11-2.02)$ \\
\hline Serum Hemoglobin $(\mathrm{g} / \mathrm{dl})$ & $-0.10(0.03)$ & $0.90(0.84-0.96)$ \\
\hline Plasma viral load $(\log 10 \mathrm{copies} / \mathrm{ml})$ & $0.22(0.04)$ & $1.25(1.14-1.36)$ \\
\hline
\end{tabular}

Table 5. Results of multivariable joint modeling of repeated measures (Global T score) and survival data

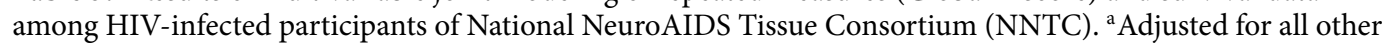
variables in the model. ${ }^{b}$ Hazard Ratio corresponds to a 10 -unit increase in global $\mathrm{T}$ scores.

rocognitive status, the association between individual neurocognitive domain and mortality was not evident among younger participants.

A sub-analysis of participants with complete data on Beck Depression Inventory-II (BDI-II) scores showed a similar association between NCI and mortality than without BDI-II in the model. For example, for participants aged 55 years, the adjusted HR was 0.83 (95\% CI 0.62-1.12) for the sub-analysis compared to 0.79 (95\% CI 0.64-0.99; Table 3) for the model without BDI-II.

Joint modeling (shared random effects model). The joint analysis estimated the individual-specific random effects of the longitudinal process simultaneously and specified them as covariates of mortality in the survival process. The linear mixed effect model generated an average regression coefficient of $0.033(\mathrm{SE}=0.003)$ (not shown) for the time variable suggesting an increase in global T scores over the study period. The linear slope estimate of the global $\mathrm{T}$ score was $-0.39(\mathrm{SE}=0.089)$, which indicates a negative association with the risk of mortality (Table 5). The adjusted HR of the slope was 0.67 (95\% CI 0.56-0.80), indicating that for every 10-unit increase in global $\mathrm{T}$ score, the hazard of death decreased by $33 \%$. The baseline covariates included in the final model (duration of HIV infection, plasma viral load, ethnicity, and serum hemoglobin) were same as those in the traditional Cox regression.

\section{Discussion}

Despite the availability and use of potent antiretroviral medications, mild to moderate HIV-associated NCI is still prevalent ${ }^{1,3}$. Apart from being a disabling consequence of HIV infection, NCI has been found to be an independent predictor of mortality in the pre-cART era ${ }^{15}$. Limited research has been conducted on the association between mortality and NCI in the HAART era, with little consideration given to the substantial variability that the course of NCI over time, in the HIV infected population ${ }^{12,15}$. The present study aimed to examine the association between baseline neurocognitive status as well as longitudinal changes in neurocognitive status and mortality in a diverse HIV-infected patient sample. Our research adds to this limited body of literature by investigating the link between HIV-associated NCI and mortality among HIV-infected participants.

The results of the crude survival analysis demonstrated no association between neurocognitive status and mortality $(\mathrm{HR}=0.99)$. In adjusted analyses we detected a significant interaction between neurocognitive status and age in relation to mortality. Specifically, among older participants, higher global T scores (i.e., better neurocognitive status) were associated with a lower hazard of death, whereas neurocognitive status was not associated with mortality among younger participants. This finding was independent of plasma viral load, ethnicity, duration of HIV infection, and serum hemoglobin as these variables were adjusted for in the final model. Unlike our study, none of the previous studies have reported an interaction between age and neurocognitive status in relation to mortality. However, studies have observed independent associations between age and mortality and age and neurocognitive status ${ }^{23,34}$. Specifically, in the pre-cART era, studies had found NCI to be an independent risk factor for mortality ${ }^{15}$. The association was also found in the cART-era, in a limited number of studies. However, the association was explored either between severe forms of NCI and mortality or only among those with the severe form of HIV infection ${ }^{25,26}$. Our findings differ from cART-era studies because we did not exclude any participants based on severity.

The most plausible mechanism underlying the interaction between age and neurocognitive status in association to mortality may be the age-related exacerbation of co-morbidities such as metabolic disorders and vascular diseases ${ }^{35-37}$. Since the initiation of cART, the prevalence of vascular and metabolic disorders among HIV infected individuals has increased because of improved life expectancy ${ }^{38}$; nearly half of people living with HIV in the United States are aged 50 and older. The synergy between age (and, indirectly, age-related comorbidities) and $\mathrm{NCI}$ in HIV probably ensues a vicious cycle. Comorbidities adversely affect the neurocognitive status, which may lead to reduced ability to manage activities of daily life, lower medication adherence, and poorer healthcare utilization, thus further progressing comorbidities ${ }^{39,40}$ Thus age may be acting as a proxy to certain comorbidities.

During domain-specific analyses, we found a stronger negative association between mortality and domainspecific T scores for abstraction and executive functioning, speed of information processing, and motor compared 
to other domains. The finding is not surprising given the recognition that HIV-related NCI tends to impact frontal-subcortical regions of the brain. To our knowledge, only one other study has conducted an analysis based on subsets of a brief screening instrument that assesses global HIV-related cognitive impairment ${ }^{27}$. Banerjee et al. used the HIV dementia scale (HDS) as a measure of NCI and assessed psychomotor speed, memory, visualspatial constructional praxis, and executive inhibitory control in relation to mortality. Among the four subset scores, executive inhibitory control (antisaccade subset) was associated with time to death, which is consistent with frontal-subcortical impairment pattern. Lastly, recent studies have found heightened variability within or between neuropsychological test scores to be associated with higher risk of death ${ }^{41,42}$. Such variability may also point to dysfunction related to attention and/or executive functions.

Apart from neurocognitive status, we also found that HIV-infected participants of non-Hispanic ethnicity, with higher viral loads, or lower hemoglobin had a higher hazard of mortality. These findings are adjusted for each other as well as for the neurocognitive status and are consistent with the literature. High plasma viral load and low serum hemoglobin are well-established independent risk factors of mortality in the HIV-infected population. Sempa et al. found a higher hazard of death per unit increase in the $\log _{10}$ viral $\operatorname{load}_{\text {( }} \mathrm{HR}=1.63 ; 95 \%$ CI 1.02-2.60 $)^{43}$, and a recent study found that viral load was a stronger predictor of mortality compared to CD4 cell count ${ }^{18}$. Lower hemoglobin has also been associated with a higher hazard (HR $=1.32,95 \%$ CI 1.12-1.55) of dying in the HIV-infected population ${ }^{44}$. Multiple studies have found a higher hazard of death among nonHispanic blacks compared to non-Hispanic whites or overall Hispanics ${ }^{45,46}$. Our findings are somewhat consistent as $72 \%$ of NNTC participants were non-Hispanics, and $46 \%$ of the non-Hispanics were blacks.

To account for the variability in the course of NCI over time and its reversible nature in relation to mortality, joint modeling was performed. Joint modeling enables the repeated NCI measurements and survival to be modeled while accounting for interrelationships between the two processes ${ }^{47}$. We found that a linear change (increase/decrease) in global T scores is an independent predictor of mortality. To our knowledge, this is the first study to examine repeated measures of NCI in relation to mortality in the HIV-infected population. Our results provide useful predictive information of longitudinal measures of NC status on mortality. The model can be used to predict future survival outcomes as well as future neurocognitive status in terms of T scores when the $\mathrm{T}$ scores are known up to the current time (dynamic prediction). Clinicians can use this information for timely detection and appropriate mitigation of NCI based on prediction.

The current study has certain limitations. This is a clinic-based study with a volunteer and predominantly male participation that may limit generalizability. This study, however, is one of its kind, conducted at four centers in the US with information available on a wide range of demographic, behavioral, and clinical and laboratory measures. The study did not exclude anyone based on the presence of other contributing central nervous system (CNS) infections; thus, the NCI may, in part, be imparted by them and not exclusively by HIV infection. However, the prevalence of other CNS infections in the study population was low (9\%). An additional limitation was that some variables were incompletely described (e.g., unclear type of hepatitis or cerebrovascular disease), or were based on self-reports (e.g., duration of HIV infection). Among the neurocognitive domains, verbal fluency was a single task domain, which may not be ideal. However, we were limited by the variables available for these secondary analyses, and this is consistent with past work by the NNTC group. Loss to follow up (12\%) in the study may have underestimated overall mortality. The final Cox proportional hazards model was not adjusted for additional confounders like depressive symptoms due to missing data. Yet, a sub-analysis of participants with complete data on Beck Depression Inventory-II (BDI-II) scores showed a similar association between NCI and mortality then without BDI-II in the model, although the results cannot be directly compared due to sample size differences. We did not investigate cause-specific mortality, as there was no relevant information available. This may have overestimated mortality in association with NCI. Furthermore, we were not able to assess the potential confounding effects of unmeasured variables, such as comorbidity treatments. Joint modeling was conducted under the assumption of a linear form for the global $\mathrm{T}$ score trajectories and censoring being independent of the random effects that may lead to model misspecifications. However, we obtained similar results with splines in the model. The positive average regression coefficient $(0.033)$ for the time variable in the longitudinal submodel within joint modelling might have been related to practice effects and not to an actual increase in global $\mathrm{T}$ scores over the study period. Practice effect is typically stronger between the between 1 st and 2 nd assessments and less evident in subsequent assessments ${ }^{48}$. Thus, we conducted a sub-analysis excluding the 1 st visit. The linear mixed effect model starting at the 2 nd visit still generated a positive average regression coefficient $(0.022)$. Lastly, repeated measures were used only for the primary exposure (global T scores), and all other covariates were assessed only at the baseline. Future research may consider including multiple covariates with repeated measures.

\section{Conclusion}

Overall, this study provides evidence that neurocognitive status interacts with age in relation to mortality and thus may have considerable prognostic utility for assessing mortality risk, particularly among older HIV-infected population. The current study identified older HIV-infected population as a group needing special attention for the longevity of life. The finding is substantial given that nearly half of people in the United States living with diagnosed HIV are aged 50 and older and that the neurocognitive status is associated with quality of life. The preliminary findings of the domain-specific analysis may suggest abstraction and executive functioning, speed of information processing, and motor domains to be particularly sensitive in relation to the hazard of mortality. The study further provides evidence that the increase in $\mathrm{T}$ scores over time is associated with lower mortality. The joint model provides useful predictive information not only about the hazard of mortality but also future cognitive scores. The study findings may be used to develop a predictive tool and thus may be used for patientspecific timely management strategies and future clinical interventions (lifestyle remedies, optimizing disease and comorbidity management and ARV management). The approach is particularly useful in clinical settings as 
repeated measures on biomarkers are very commonly generated for monitoring of chronic medical conditions. In conclusion, optimal management (treatment/rehabilitation) strategies, particularly based on the dynamic predictions and targeting not only the neurocognitive status but also age-related co-morbidities, may lead to an improved outcome in the HIV-infected population.

Received: 23 August 2020; Accepted: 19 January 2021

Published online: 12 February 2021

\section{References}

1. Antinori, A. et al. Updated research nosology for HIV-associated neurocognitive disorders. Neurology 69, 1789-1799 (2007).

2. De Cock, K. M., Jaffe, H. W. \& Curran, J. W. The evolving epidemiology of HIV/AIDS. AIDS 26, 1205-1213 (2012).

3. Heaton, R. K. et al. HIV-associated neurocognitive disorders persist in the era of potent antiretroviral therapy: CHARTER Study. Neurology 75, 2087-2096 (2010).

4. Cysique, L. A. \& Brew, B. J. Prevalence of non-confounded HIV-associated neurocognitive impairment in the context of plasma HIV RNA suppression. J. Neurovirol. 17, 176-183 (2011).

5. Mind Exchange Working Group et al. Assessment, diagnosis, and treatment of HIV-associated neurocognitive disorder: A consensus report of the mind exchange program. Clin. Infect. Dis. 56, 1004-1017 (2013).

6. Lindl, K. A., Marks, D. R., Kolson, D. L. \& Jordan-Sciutto, K. L. HIV-associated neurocognitive disorder: Pathogenesis and therapeutic opportunities. J. Neuroimmune Pharmacol. 5, 294-309 (2010).

7. Albright, A. V., Soldan, S. S. \& González-Scarano, F. Pathogenesis of human immunodeficiency virus-induced neurological disease. J. Neurovirol. 9, 222-227 (2003).

8. Lentz, M. R. et al. Changes in MRS neuronal markers and T cell phenotypes observed during early HIV infection. Neurology 72 , 1465-1472 (2009).

9. Woods, S. P. et al. Interrater reliability of clinical ratings and neurocognitive diagnoses in HIV. J. Clin. Exp. Neuropsychol. 26, 759-778 (2004).

10. Saylor, D. et al. HIV-associated neurocognitive disorder-pathogenesis and prospects for treatment. Nat. Rev. Neurol. 12, 234 (2016).

11. Ances, B. M. et al. Resting cerebral blood flow: A potential biomarker of the effects of HIV in the brain. Neurology 73, 702-708 (2009).

12. McArthur, J. C. HIV dementia: An evolving disease. J. Neuroimmunol. 157, 3-10 (2004).

13. Letendre, S. L., Ellis, R. J., Ances, B. M. \& McCutchan, J. A. Neurologic complications of HIV disease and their treatment. Top. HIV. Med. 18, 45-55 (2010).

14. Heaton, R. K. et al. HIV-associated neurocognitive disorders before and during the era of combination antiretroviral therapy: Differences in rates, nature, and predictors. J. Neurovirol. 17, 3-16 (2011).

15. Ellis, R. J. et al. Neurocognitive impairment is an independent risk factor for death in HIV infection. Arch. Neurol. 54, 416-424 (1997).

16. van Sighem, A. et al. Mortality in patients with successful initial response to highly active antiretroviral therapy is still higher than in non-HIV-infected individuals. JAIDS 40, 212-218 (2005).

17. Bhaskaran, K. et al. Changes in the risk of death after HIV seroconversion compared with mortality in the general population. JAMA 300, 51-59 (2008).

18. Shoko, C. \& Chikobvu, D. A superiority of viral load over CD4 cell count when predicting mortality in HIV patients on therapy. BMC Infect. Dis. 19, 169 (2019).

19. Adams, J. W. et al. Association of cannabis, stimulant, and alcohol use with mortality prognosis among HIV-infected men. AIDS. Behav. 22, 1341-1351 (2018).

20. Brothers, T. D. et al. Predictors of transitions in frailty severity and mortality among people aging with HIV. PLoS One 12, e0185352 (2017).

21. Wilkie, F. L. et al. Mild cognitive impairment and risk of mortality in HIV-1 infection. J. Neuropsychiatry Clin. Neurosci. 10, 125-132 (1998).

22. Mayeux, R. et al. Mortality risks in gay men with human immunodeficiency virus infection and cognitive impairment. Neurology. 43, 176-182 (1993).

23. Patel, S. et al. United States National Trends in mortality, length of stay (LOS) and associated costs of cognitive impairment in HIV population from 2005 to 2014. AIDS Behav. 22, 3198-3208 (2018).

24. Dore, G. J. et al. Marked improvement in survival following AIDS dementia complex in the era of highly active antiretroviral therapy. AIDS 17, 1539-1545 (2003).

25. Lescure, F. et al. Incidence and impact on mortality of severe neurocognitive disorders in persons with and without HIV infection: A Danish nationwide cohort study. Clin. Infect. Dis. 52, 235-243 (2011).

26. Sevigny, J. J. et al. An evaluation of neurocognitive status and markers of immune activation as predictors of time to death in advanced HIV infection. Arch. Neurol. 64, 97-102 (2007).

27. Banerjee, N., McIntosh, R. C. \& Ironson, G. Impaired neurocognitive performance and mortality in HIV: Assessing the prognostic value of the HIV-dementia scale. AIDS Behav. 23, 3482-3492 (2019).

28. Hu, X. et al. Diagnostic accuracy of the international HIV dementia scale and HIV dementia scale: A meta-analysis. Exp. Ther. Med. 4, 665-668 (2012).

29. Bottiggi, K. A. et al. The HIV Dementia Scale: Predictive power in mild dementia and HAART. J. Neurol. Sci. 260, 11-15 (2007).

30. NNTC. National NeuoAIDS Tissue Consortium-About. https://nntc.org/index. Accessed 26 Jan 2020.

31. Morgello, S. et al. The National NeuroAIDS Tissue Consortium: A new paradigm in brain banking with an emphasis on infectious disease. Neuropathol. Appl. Neurobiol. 27, 326-335 (2001).

32. Heithoff, A. J. et al. The integrated National NeuroAIDS Tissue Consortium database: A rich platform for neuroHIV research. Database 20, 20 (2019).

33. Cassol, E., Misra, V., Morgello, S. \& Gabuzda, D. Applications and limitations of inflammatory biomarkers for studies on neurocognitive impairment in HIV infection. J. Neuroimmune Pharmacol. 8, 1087-1097 (2013).

34. Ding, Y. et al. Interaction effects between HIV and aging on selective neurocognitive impairment. J. Neuroimmune Pharmacol. 12, 661-669 (2017).

35. Cohen, R. A., Seider, T. R. \& Navia, B. HIV effects on age-associated neurocognitive dysfunction: Premature cognitive aging or neurodegenerative disease?. Alzheimers Res. Ther. 7, 37 (2015).

36. Currier, J. S. et al. Epidemiological evidence for cardiovascular disease in HIV-infected patients and relationship to highly active antiretroviral therapy. Circulation 118, e29-e35 (2008).

37. Cruse, B., Cysique, L. A., Markus, R. \& Brew, B. J. Cerebrovascular disease in HIV-infected individuals in the era of highly active antiretroviral therapy. J. Neurovirol. 18, 264-276 (2012). 
38. Collaboration, A. T. C. Life expectancy of individuals on combination antiretroviral therapy in high-income countries: A collaborative analysis of 14 cohort studies. Lancet 372, 293-299 (2008).

39. Ettenhofer, M. L. et al. Aging, neurocognition, and medication adherence in HIV infection. Am. J. Geriatr Psychiatry 17, 281-290 (2009).

40. Morgan, E. E. et al. Neurocognitive impairment is associated with lower health literacy among persons living with HIV infection. AIDS Behav. 19, 166-177 (2015).

41. MacDonald, S. W., Hultsch, D. F. \& Dixon, R. A. Predicting impending death: Inconsistency in speed is a selective and early marker. Psychol. Aging. 23, 595 (2008).

42. Anderson, A. E. et al. Intraindividual variability in neuropsychological performance predicts cognitive decline and death in HIV. Neuropsychology 32, 966 (2018).

43. Sempa, J. B. et al. Reevaluating cumulative HIV-1 viral load as a prognostic predictor: Predicting opportunistic infection incidence and mortality in a Ugandan Cohort. Am. J. Epidemiol. 184, 67-77 (2016).

44. Bisson, G. P. et al. Risk factors for early mortality on antiretroviral therapy in advanced HIV-infected adults. AIDS 31, 2217-2225 (2017).

45. Woldemichael, G., Christiansen, D., Thomas, S. \& Benbow, N. Demographic characteristics and survival with AIDS: Health disparities in Chicago, 1993-2001. Am. J. Public Health. 99, S118-S123 (2009).

46. Trepka, M. J. et al. Sex and racial/ethnic differences in premature mortality due to HIV: Florida, 2000-2009. Public Health Rep. 130, 505-513 (2015).

47. Lim, H., Mondal, P. \& Skinner, S. Joint modeling of longitudinal and event time data: Application to HIV study. J. Med. Stat. Inform. $1,1(2013)$.

48. Collie, A., Maruff, P., Darby, D. G. \& McSthephen, M. The effects of practice on the cognitive test performance of neurologically normal individuals assessed at brief test-retest intervals. J. Int. Neuropsychol. Soc. 9, 419 (2003).

49. Heaton, R., Miller, S. W., Taylor, M. J. \& Grant, I. Revised Comprehensive Norms for an Expanded Halstead-Reitan Battery: Demographically Adjusted Neuropsychological Norms for African American and Caucasian Adults (Psychological Assessment Resources, Lutz, 2004).

50. Norman, M. A. et al. Demographically corrected norms for African Americans and Caucasians on the Hopkins verbal learning test-revised, brief visuospatial memory test-revised, Stroop color and word test, and Wisconsin card sorting test 64-card version. J. Clin. Exp. Neuropsychol. 33, 793-804 (2011).

51. David, W. Wechsler Adult Intelligence Scale (Psychological Corporation, San Antonio, 1997).

52. Diehr, M. C. et al. The 50 and 100-item short forms of the Paced Auditory Serial Addition Task (PASAT): Demographically corrected norms and comparisons with the full PASAT in normal and clinical samples. J. Clin. Exp. Neuropsychol. 25, 571-585 (2003).

53. Benedict, R. H. Brief Visuospatial Memory Test-Revised (PAR, 1997).

54. Benedict, R. H., Schretlen, D., Groninger, L. \& Brandt, J. Hopkins verbal learning test-revised: Normative data and analysis of inter-form and test-retest reliability. Clin. Neuropsychol. 12, 43-55 (1998).

\section{Author contributions}

Conceptualization and designing of the study were performed by Z.N., H.S.F., L.B., P.M., C.M.A. and S.T. Data was acquired by H.S.F. and S.T. Data analysis was performed by Z.N., L.B., C.S.W., J.M., M.A. and S.T. Manuscript was drafted by Z.N., L.B. and P.M. Review and revision of article was performed by Z.N., H.S.F., L.B., C.S.W., M.A., P.M., C.M.A., J.M. and S.T. All authors have read the manuscript and approved its contents.

\section{Funding}

Supported by the National Institutes of Health (NIH) under Award Number U24MH100925. The content is solely the responsibility of the authors and does not necessarily represent the official views of the NIH or NNTC. Support for the NNTC is provided by the National Institutes of Health (NIH) under Award Numbers U24MH100925 U24MH100928, U24MH100929, U24MH100930, and U24MH100931.

\section{Competing interests}

The authors declare no competing interests.

\section{Additional information}

Supplementary Information The online version contains supplementary material available at https://doi. org/10.1038/s41598-021-83131-1.

Correspondence and requests for materials should be addressed to L.B.

Reprints and permissions information is available at www.nature.com/reprints.

Publisher's note Springer Nature remains neutral with regard to jurisdictional claims in published maps and institutional affiliations.

Open Access This article is licensed under a Creative Commons Attribution 4.0 International License, which permits use, sharing, adaptation, distribution and reproduction in any medium or format, as long as you give appropriate credit to the original author(s) and the source, provide a link to the Creative Commons licence, and indicate if changes were made. The images or other third party material in this article are included in the article's Creative Commons licence, unless indicated otherwise in a credit line to the material. If material is not included in the article's Creative Commons licence and your intended use is not permitted by statutory regulation or exceeds the permitted use, you will need to obtain permission directly from the copyright holder. To view a copy of this licence, visit http://creativecommons.org/licenses/by/4.0/.

(C) The Author(s) 2021 